\title{
Nano-FTIR Spectroscopy of Intersubband Polaritons in Single Nanoantenna
}

\author{
C.-F. Wang, ${ }^{1,2}$ T. G. Habteyes, ${ }^{1}$ T. S. Luk, ${ }^{3,4}$ J. F. Klem, ${ }^{4}$ I. Brener, ${ }^{3,4}$ H.-T. Chen, ${ }^{2}$ and O. Mitrofanov ${ }^{1,2,3}$ \\ ${ }^{1}$ Center for High Technology Materials, University of New Mexico, Albuquerque, NM 87106, USA \\ ${ }^{2}$ Center for Integrated Nanotechnologies, Los Alamos National Laboratory, Los Alamos, NM 87545, USA \\ ${ }^{3}$ Center for Integrated Nanotechnologies, Sandia National Laboratories, Albuquerque, New Mexico 87123, USA \\ ${ }^{4}$ Sandia National Laboratories, Albuquerque New Mexico 87185, USA \\ ${ }^{5}$ Electronic and Electrical Engineering, University College London, London, WC1E 7JE United Kingdom
}

\begin{abstract}
We demonstrate observation of infrared (IR) intersubband (ISB) polaritons in an isolated subwavelength size nanoantenna using near-field Fourier-transform infrared (FTIR) spectroscopy of the evanescent fields on the nanoantenna surface. The near-field approach enables detection of the distinctive polariton splitting of the nanoantenna resonance in the amplitude and phase spectra, as well as mapping of the ISB polariton dispersion. The nano-FTIR spectroscopy approach opens doors for investigations of light-matter interaction in the single subwavelength nanoantenna regime.
\end{abstract}

\section{INTRODUCTION}

$\mathrm{S}_{\mathrm{q}}$ TRONG coupling of an intersubband (ISB) transition in (1) a subwavelength plasmonic nanoantenna [1] enables a range of practical devices, such as non-linear metasurfaces and low threshold lasers [2]. It can also lead to exotic quantum phenomena, such as ISB polariton condensation [3]. For studies of quantum phenomena, it is desirable to work with ISB polaritons in an isolated subwavelength-size system, for instance a single nanoscale antenna. Observation of ISB polaritons in a single nanoantenna coupled to the ISB transition however has not yet been reported, because a small extinction cross section makes far-field experiments on a single subwavelength antenna challenging $[4,5]$. Near-field microscopy offers an alternative approach to probe the interaction of a subwavelength antenna with the ISB system. Here we employ a scattering-type scanning near-field optical microscope (s-SNOM) and enable ISB polariton studies in the single nanoantenna regime [Fig. 1(a)].

\section{RESULTS}

The ISB transition investigated here is between the ground and excited states in a $9.5 \mathrm{~nm}$ thick doped quantum well made of $\mathrm{In}_{0.53} \mathrm{Ga}_{0.47} \mathrm{As}$ with $\mathrm{Al}_{0.48} \mathrm{In}_{0.52} \mathrm{As}$ barriers. Our sample contains a stack of 22 quantum wells. The ISB transition energy estimated numerically is $\sim 1100 \mathrm{~cm}^{-1}$. Using transmission FTIR spectroscopy in the waveguide configuration we experimentally find that the transition energy is $\sim 1150 \mathrm{~cm}^{-1}$.

We fabricate a range of dogbone shaped nanoantennas of different sizes directly on the semiconductor surface [Fig. 1(b)]. The nanoanennae provide subwavelength field confinement of the resonant fields in the region of quantum wells. For an antenna of length $l=950 \mathrm{~nm}$, we estimate that the dipolar resonance is at $\lambda_{\mathrm{c}} \sim 10 \mu \mathrm{m}$, using numerical modeling.

Although the dipolar mode for the dogbone antenna has strong sub-surface confinement, a small amount of the mode energy tends to leak to the nanoantenna top surface via surface waves [6]. This suggests that it is possible to detect ISB polaritons using near-field microscopy. We probe evanescent (a)
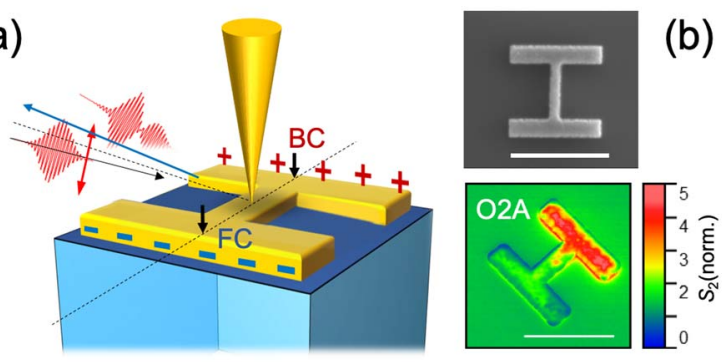

(c)
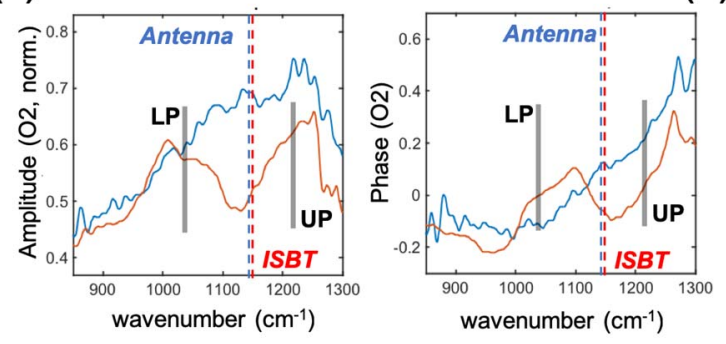

(d)

Fig. 1. Nano-FTIR spectroscopy of nanoantennae coupled to the ISB transition in quantum wells in the substrate underneath the antenna. (a) Schematic diagram of the experimental setup: for nano-FTIR analysis, the probe is positioned either at the center of the front (FC) or the back (BC) bars (marked by black arrows). (b) SEM image (top) and near-field amplitude image (bottom) of the $l=950 \mathrm{~nm}$ antenna in resonance with excitation at $1000 \mathrm{~cm}^{-1}$. The scale bar is $1 \mu \mathrm{m}$. FTIR amplitude (c) and phase (d) of the evanescent filed at the back bar of the $l=850 \mathrm{~nm}$ nanoantenna in resonance with the ISBT transition at $1150 \mathrm{~cm}^{-1}$ (red lines). For comparison, blue lines show amplitude and phase spectra of a nanoantenna of the same size fabricated on a different sample with the ISB transition at $815 \mathrm{~cm}^{-1}$, outside the spectral range of the excitation pulse.

fields on the surface of an isolated nanoantenna using a commercial s-SNOM system (neaSpec GmbH). First, we excite a nanoanenna with CW IR laser light at the frequency of 1000 $\mathrm{cm}^{-1}$. The laser beam is polarized in the plane of incidence. Although the dipolar mode at this frequency is distributed mainly underneath the surface, within the depth of $\sim 500 \mathrm{~nm}$, the evanescent field on the antenna surface shows a dipolar spatial distibution, confirming that it is possible to detect the antenna mode in the amplitude of the enanescent filed on the surface [Fig. 1(b), bottom].

Next we excite the nanoantenna by a short IR pulse $\left(\lambda_{\mathrm{c}} \sim 10\right.$ $\left.\mu \mathrm{m}, 850-1300 \mathrm{~cm}^{-1}\right)$ [Fig. 1(a)], and spectrally analyze the evanescet field on the dogbone antenna bars. The near-field probe scatters a small fraction of the evanescent field, which we combine with a reference IR pulse for coherent FTIR spectroscopy. By scanning temporal delay between the interfering pulses, we obtain a cross-correlation interferogram, 
from which we exract the amplitude and phase of the evanescent field on the antenna surface. We find that the evanescent field exhibits a spectral peak at the resonance frequency of the antenna [7].

Next, we select an antenna with the resonance at the frequency of the ISB transition $\left(1150 \mathrm{~cm}^{-1}\right)$. Results of nanoFTIR spectroscopy analysis for this antenna $(l=850 \mathrm{~nm})$ are shown in Fig. 1(c,d). Instead of the antenna resonance peak we observe splitting of the antenna resonance into two distict ISB polariton modes above and below the antenna frequency, indicative of strong coupling of the antenna field to the ISB transition in the quantum wells [5,7]. For comparison, Fig. 1 shows also nano-FTIR spectra of a nanoantenna with the same dimensions, but fabricated on a sample with wider quantum wells, which exhibit the ISB transision at $815 \mathrm{~cm}^{-1}$. That antenna shows only a single peak at $\sim 1150 \mathrm{~cm}^{-1}$. Confirming that the splitting is indeed due to the coupling of the antenna mode with the ISB transition.

The nano-FTIR analysis also provides the phase of the evanescent fields on the surface. We find that in the case of two polariton peaks, the phase spectrum exhibits two steplike changes at the frequencies of the lower and upper polaritons, whereas the the phase spectrum of the nanoantenna without ISB transition coupling exhibits only one step at the antenna resonance frequency. Detailed analysis of the phase spectra are discussed in Ref. 7.

We now map dispersion characteristics of the nanoantennaISB system in the single nanoantenna regime. We fabricate a set of nanoantennae with size $l$ ranging from $600 \mathrm{~nm}$ to 1600

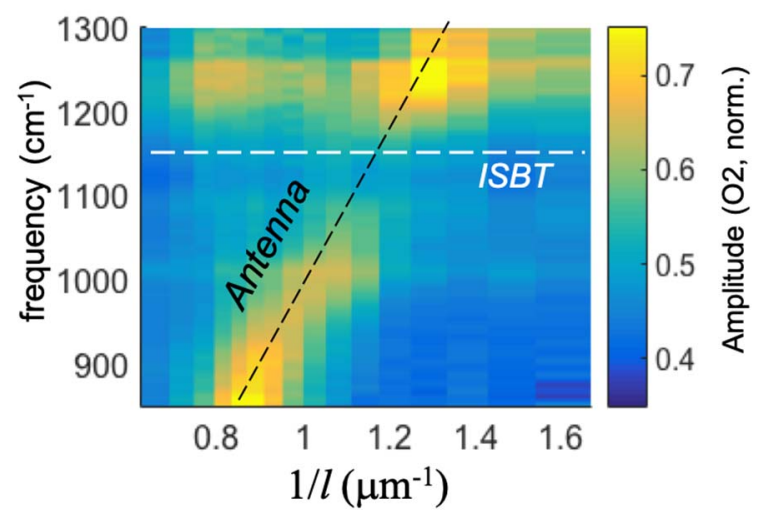

Fig. 2. Spectral amplitude map of the evanescent field at the back bar of the nanoantenna measured using a set of nanoantennae with lengths $l$ ranging from $600 \mathrm{~nm}$ to $1600 \mathrm{~nm}$. The antenna dispersion is shown by the black dashed line, and the ISB transition at $1150 \mathrm{~cm}^{-1}$ is marked by the white dashed line.

$\mathrm{nm}$, and record nano-FTIR amplitude and phase spectra at the same point on the back bar for every antenna. It allows us to "tune" the dipolar resonance across the ISB transition and map the dispersion properties of the ISB polaritons. We find that the amplitude of the resonant antenna field is suppressed at the antenna resonance frequency, and instead the ISB-nanoantenna system exhibits a mode anti-crossing behavior with the mode splitting of $\sim 190-200 \mathrm{~cm}^{-1}$ [Fig. 2].

\section{SUMMARY}

We demonstrate nano-FTIR spectroscopy of ISB polaritons in a single isolated nanoantenna in the IR frequency range. This approach opens doors to a range of studies of ISB polaritons in the single nanoantenna regime, e.g. investigations of the elusive effect of ISB polariton condensation. We anticipate that this near-field approach will also enable explorations of strong and ultrastrong light-matter coupling in the single nanoantenna regime for other excitations

\section{ACKNOWLEDGEMENTS}

This work was supported by the U.S. Department of Energy, Office of Basic Energy Sciences, Division of Materials Sciences and Engineering. Fabrication and nano-FTIR measurements were performed at the Center for Integrated Nanotechnologies, an Office of Science User Facility operated for the U.S. Department of Energy (DOE) Office of Science. Sandia National Laboratories is a multi-mission laboratory managed and operated by National Technology and Engineering Solutions of Sandia, LLC., a wholly owned subsidiary of Honeywell International, Inc., for the U.S. Department of Energy's National Nuclear Security Administration under contract DE-NA-0003525. Los Alamos National Laboratory, an affirmative action equal opportunity employer, is managed by Triad National Security, LLC for the U.S. Department of Energy's NNSA, under contract 89233218CNA000001. This article describes objective technical results and analysis. The views expressed in the article do not necessarily represent the views of the U.S. DOE or the United States Government. C.-F. W and T. H. were supported in part by the U.S. National Science Foundation (Grant No. 1651478), and O.M. by EPSRC.

\section{REFERENCES}

[1]. Y. Todorov and C. Sirtori, Phys. Rev. B 85, 045304 (2012).

[2]. A. F. Kockum, A. Miranowicz, S. De Liberato, S. Savasta and F. Nori, Nat. Rev. Phys. 1, 19-40 (2019).

[3]. R. Colombelli and J.-M. Manceau, Phys. Rev X 5, 011031 (2015).

[4]. M. Malerba, T. Ongarello, B. Paulillo, J.-M. Manceau, G. Beaudoin, I. Sagnes, F. De Angelis, and R. Colombelli, Appl. Phys. Lett. 109, 021111 (2016)

[5]. A. Benz, S. Campione, S. Liu, I. Montano, J.F. Klem, A. Allerman, J.R. Wendt, M.B. Sinclair, F. Capolino, and I. Brener, Nat. Commun. 4, 2882 (2013).

[6]. O. Mitrofanov, Y. Todorov, D. Gacemi, A. Mottaghizadeh, C. Sirtori, I. Brener, J.L. Reno, Opt. Express 26, 7437-7450 (2018).

[7]. C.-F. Wang, T. G. Habteyes, T. S. Luk, J. F. Klem, I. Brener, H.-T. Chen, and O. Mitrofanov, Nano Lett. (published online, DOI: 10.1021/acs.nanolett.9b01623, 2019). 